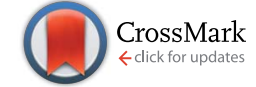

Cite this: RSC Adv., 2017, 7, 8898

Received 12th December 2016 Accepted 22nd January 2017

DOI: 10.1039/c6ra28060a

rsc.li/rsc-advances

\section{Tailoring the alignment of string-like nanoparticle assemblies in a functionalized polymer matrix via steady shear}

\begin{abstract}
Yulong Chen, ${ }^{\star a}$ Jun Liu, ${ }^{\mathrm{b}} \mathrm{Li}$ Liu, ${ }^{\mathrm{b}}$ Huanre Han, ${ }^{\mathrm{a}}$ Qian Xu${ }^{\mathrm{a}}$ and Xin Qian*a
Recent work has shown that nanoparticles (NPs) dispersed in a sequence specific functionalized polymer matrix can be organized into a variety of superstructures, such as strings, sheets, and percolated networks. However, these spontaneously formed structures are generally ordered within the short range. To exploit the use of NPs to their full potential, defect-free, long-range ordered NP organization is usually necessary. The use of a shear field may be an appropriate means to reach this objective due to its well-understood orientation effect. Therefore, the present study seeks to probe the effect of steady shear on the ordering of string-like NP assemblies in a functionalized polymer matrix on the basis of coarse-grained molecular dynamics simulations. We found that by properly tuning shear rate, the shear can induce one-dimensional alignment to the NP strings, and the emerging structures can remain stable after the cessation of shear, which is required for many applications such as the fabrication of nanomaterial arrays. The mechanism for forming such a well-defined structure can be interpreted from the competition among the NP mobility, polymer relaxation, NP-functional group interaction, and external shear effect. It may be expected that the information derived in the present study provides a useful foundation for guiding the design and preparation of tailored PNCs.
\end{abstract}

\section{Introduction}

Controlling the organization of nanoparticles (NPs) in polymer matrices with high precision has received tremendous interest since the collective properties of polymer nanocomposites (PNCs) significantly depend on the dispersion and spatial arrangement of NPs. ${ }^{1,2}$ It has been shown that some PNC properties are optimized when the NPs are randomly dispersed, ${ }^{3}$ while others require the NPs to form percolated networks ${ }^{4,5}$ or to align in a given direction. ${ }^{6,7}$ A number of routes have been developed to achieve these goals. For NP anisotropic assembly, the most direct strategy is to use NPs with anisotropic shapes or directional interactions. ${ }^{8-12}$ It was generally believed that it is difficult for isotropically interacting spherical NPs to self-assemble into anisotropic superstructures. However, recently, some studies have established approaches for creating a range of anisotropic structures from common spherical NPs. ${ }^{13-18}$ As a typical representative, Kumar's group ${ }^{13,19-22}$ found that spherical NPs uniformly grafted with polymer chains can self-assemble into strings and sheets.

Similar to the findings of Kumar's group, some studies have also demonstrated that bare spherical NPs dispersed in

${ }^{a}$ College of Materials Science and Engineering, Zhejiang University of Technology, Hangzhou 310014, China.E-mail: chenyulong@zjut.edu.cn; qianx@zjut.edu.cn

${ }^{b}$ Beijing Engineering Research Center of Advanced Elastomers, Beijing University of Chemical Technology, Beijing 100029, China sequence specific functionalized polymers ${ }^{23-25}$ can be organized into a variety of morphologies, where the backbones of the functionalized chains inherently dislike the NPs but the periodically spaced functional groups on the chains are designed to show specific affinity for the NPs. ${ }^{17,18}$ The adsorption of the functional groups on the NP surfaces makes the NPs act like transient "grafted NPs" (Fig. 1d). The dislike between the "grafts" and NP cores drives the self-assembly, which is similar to the behaviour of microphase-separated block copolymers. ${ }^{26}$ When the polymer chains are highly functionalized, the NPs are well dispersed due to steric (entropic) repulsion of the dense surrounding "grafts", that is, the attractions between the NPs (including van der Waals and depletion forces) are strongly reduced by the "grafts". In the opposite limit of low functionalization, the NPs aggregate, owing to the dominance of the NPNP attractions. Intermediate values of functionalization degree yield a variety of interconnected NP structures such as strings, sheets, and networks, due to a balance of the NP-NP attractions and the steric stabilization of the "grafts" which leads to anisotropic interactions. Among these cases, the string-like NP assemblies gained considerable interest because NPs organized in this way behave akin to "nanowires" ${ }^{\text {"7,28 }}$ which is advantageous for transport or conductive properties of PNCs. However, these spontaneously formed structures are generally ordered within the short-range, but disordered on a larger scale, due to the isotropic phase structure of the polymer matrices. 


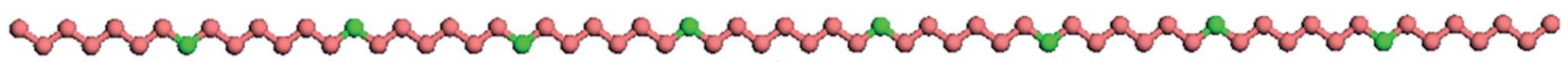

(a)
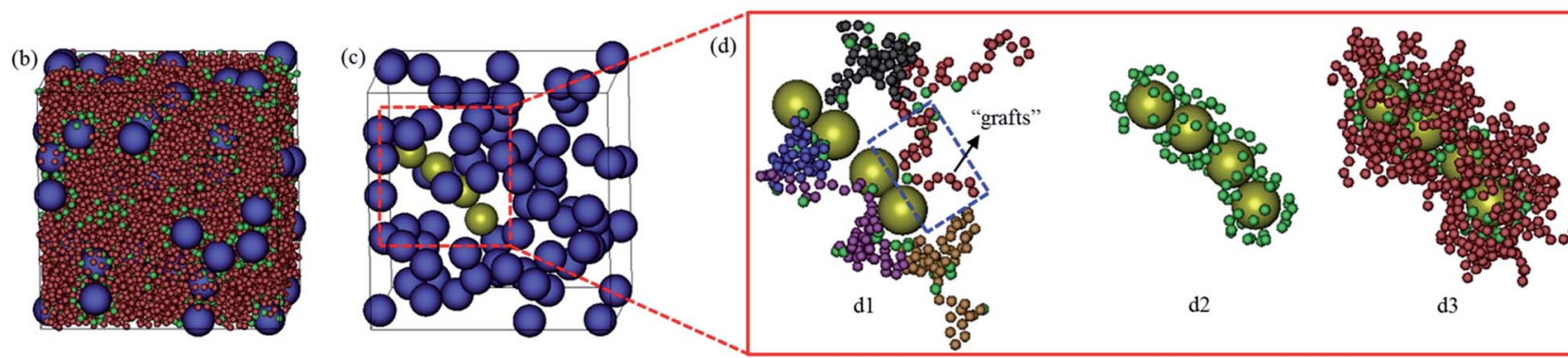

d3

Fig. 1 (a) Coarse-grained model of in-chain functionalized polymer, where the functionalized and unfunctionalized beads are shown as green and red spheres, respectively. ( $\mathrm{b}$ and c) Snapshots for the quiescent system with functionalization degree $d_{\mathrm{F}}=0.12$ and filler volume fraction $\phi_{\mathrm{NP}}$ $=0.15$. (d) Close-up views of a short NP string extracted from (c). The short string is colored yellow while the remaining NPs are shown in blue color. For presentation purposes, in (c) we omit the polymer matrix, in (d1) we illustrate five typical chains (in different colors) bridged to the string through their adsorbing (functional) sites, in (d2) we show the functionalized beads adsorbed on the string, and in (d3) we depict the polymer "grafts" (of length of six beads) connected to the adsorbed functionalized beads.

For some anisotropic functional PNCs, long-range order of NPs in a given direction is usually needed. To reach this objective, the use of a shear field may be an appropriate approach due to its well-understood orientation effect. ${ }^{29,30}$ Compared to the alternative strategies, e.g., electric and magnetic fields, the advantage of shear flow is the ability to order microstructures over macroscopic length scales and no need for doping or exotic NP properties, which makes the shear alignment feasible for large-scale industrial manufacturing.

Investigating the shear behaviour of NPs in polymers via experiments is often prohibitively costing and time-consuming. Meanwhile, the experimental techniques still have a great challenge in revealing the microscopic morphologies of NPs under flow conditions. Computer simulations have emerged as useful techniques in addressing these issues. ${ }^{31-33}$ Several methods have been developed for simulations of PNC systems. Atomistic or near-atomistic simulations are quite accurate approaches to understand the structural and dynamic properties of PNCs at the molecular level, ${ }^{34-36}$ but only allow for the sampling of relatively small systems and short time scales, since they are rather computationally expensive. The length- and time-scale limitations of these methods are particularly restrictive for the study of the NP dispersion or organization in polymer melts under shear. On the other hand, the phase-field methods such as Cahn-Hilliard ${ }^{37}$ and self-consistent field theories ${ }^{38,39}$ enable simulation over long time scales and for large systems, but prohibit the detailed analysis of microscopic properties. For these reasons, coarse-grained models which group atoms or molecules into single particles have been widely adopted to investigate the sheared PNC systems. ${ }^{40-52}$ Although these models are of much lower resolution than atomistic, they still can describe the microscopic nature of the systems at length scales comparable to the intermolecular distances. However, up to now, there still no study related to the investigation of functionalized polymer/NP systems under shear.

In this study, we seek to employ coarse-grained molecular dynamics (CGMD) simulations to investigate the steady-shear behaviour of string-like NP assemblies in functionalized polymers. We begin by studying how shear rate affects the ordering of the NP strings and find that there exists an optimal shear-rate range to induce the formation of stabilized one-dimensional (1D) alignment of NP strings. Such well-defined structure can also be observed in other similar systems. We then discuss the structural formation mechanism based on the structural, dynamic, and rheological properties of the equilibrated and sheared PNC systems. Finally, the relaxing behaviour of these systems after ceasing shear is also considered to explore the stability of the shear-induced aligned NP strings.

\section{Computational methods}

\subsection{Coarse-grained models}

Our model PNC systems (Fig. 1b) are composed of bare spherical NPs (Fig. 1c) within linear in-chain functionalized polymers (Fig. 1a). The functionalized polymer chains are modelled using the standard bead-spring (Kremer-Grest) model ${ }^{53}$ with $N=65$ spherical beads of size $\sigma$ and mass $m$. Each chain consists of two types of beads, "B" and " $F$ ". B beads are backbone beads, while $\mathrm{F}$ beads are functionalized beads which are uniformly distributed within the polymer chain (Fig. 1a). The functionalization degree of the polymer was defined as $d_{\mathrm{F}}=N_{\mathrm{F}} / N$, where $N_{\mathrm{F}}$ being the number of functionalized beads in a chain. The adjacent beads in each chain are connected via a strong finitely extensible nonlinear elastic (FENE) potential:

$$
U_{\mathrm{FENE}}(r)=-\frac{k}{2} R_{0}^{2} \ln \left[1-\left(\frac{r}{R_{0}}\right)^{2}\right]
$$

where $r$ is the distance between bonded beads, $k=30 \varepsilon / \sigma^{2}$ is the spring constant, $R_{0}=1.5 \sigma$ is the maximum possible length of the spring. The nonbonded interactions between all polymer beads, both intra- and intermolecular, are described using a short-range purely repulsive potential, also known as the Weeks-Chandler-Anderson (WCA) potential: ${ }^{54}$ 


$$
U_{\mathrm{WCA}}(r)=\left\{\begin{array}{cc}
4 \varepsilon\left[\left(\frac{\sigma}{r}\right)^{12}-\left(\frac{\sigma}{r}\right)^{6}+\frac{1}{4}\right] & r<2^{1 / 6} \sigma \\
0 & r \geq 2^{1 / 6} \sigma
\end{array}\right.
$$

where $r$ is the distance between the nonbonded beads.

The NPs are treated as spheres of diameter $\sigma_{\mathrm{NP}}=4 \sigma$ and mass $m_{\mathrm{NP}}=64 m$ that are weakly attractive with each other. The interactions between the NPs and B beads are purely repulsive, while the interactions between the NPs and F beads are strongly attractive. These interactions are modeled via the truncated and shifted Lennard-Jones (LJ) potential:

$$
\begin{aligned}
U_{\mathrm{NP}-\mathrm{X}}^{\mathrm{LJ}}(r)= & 4 \varepsilon_{\mathrm{NP}-\mathrm{X}}\left[\left(\frac{\sigma}{r-r_{\mathrm{ev}}}\right)^{12}-\left(\frac{\sigma}{r-r_{\mathrm{ev}}}\right)^{6}-\left(\frac{\sigma}{r_{\mathrm{c}}-r_{\mathrm{ev}}}\right)^{12}\right. \\
& \left.+\left(\frac{\sigma}{r_{\mathrm{c}}-r_{\mathrm{ev}}}\right)^{6}\right]
\end{aligned}
$$

where $U_{\mathrm{NP}-\mathrm{X}}^{\mathrm{LJ}}(r)=0$ for $r-r_{\mathrm{ev}}>r_{\mathrm{c}}$, the subscript $X \in\{\mathrm{NP}, \mathrm{B}, \mathrm{F}\}$, $\varepsilon_{\mathrm{NP}-\mathrm{X}}$ is the interaction strength (LJ well depth), $r_{\mathrm{ev}}$ is adjustable parameter to account for the excluded volume effects of different interacting sites, and $r_{\mathrm{c}}$ is the cutoff distance of the potential. The potential parameters are summarized in Table 1. It should be noted that when mapping the bead-spring model to real polymers, the energy scale $\varepsilon$ is about $2.5-4.2 \mathrm{~kJ} \mathrm{~mol}^{-1} .{ }^{53} \mathrm{It}$ has been reported that the surface of carbon black, a traditional reinforcing filler for rubber, is energetically very heterogeneous, consisting of different adsorption sites from 16 to $30 \mathrm{~kJ} \mathrm{~mol}^{-1} .^{55}$ The interaction strengths would be much stronger when the acidic groups on the surface of carbon black, such as carboxyl, phenol, and hydroxyl, interact with functional groups of functionalized polymers to form ionic bonds or hydrogen bonds. ${ }^{24}$ Thus, the interaction strength of $\varepsilon_{\mathrm{NP}-\mathrm{F}}=10 \varepsilon$ (about 25-42 kJ $\mathrm{mol}^{-1}$ ) is appropriate to mimic the strong attraction between the NPs and functional groups in real PNC systems.

\subsection{Simulation details}

In this work, all the physical quantities are expressed in terms of $\mathrm{LJ}$ reduced units where $\sigma, m$, and $\varepsilon$ are the basic length, mass, and energy scales, respectively. The reduced temperature and time units are defined as $T=\varepsilon / k_{\mathrm{B}}$ (where $k_{\mathrm{B}}$ is the Boltzmann constant) and $\tau=\sigma(m / \varepsilon)^{1 / 2}$, respectively.

We carried out the MD simulations using the open source LAMMPS package developed by Sandia National Laboratories, ${ }^{56}$ where the equations of motion are integrated using a velocityVerlet algorithm with a time step of $\Delta t=0.001 \tau$. The temperature was kept fixed at $T=\varepsilon / k_{\mathrm{B}}$ via a Nosé-Hoover thermostat.

Table 1 Potential parameters

\begin{tabular}{lccc}
\hline Label & NP-NP & & \\
\hline$\varepsilon_{\mathrm{NP}-\mathrm{X}}$ & $1.0 \varepsilon$ & $\mathrm{NP}^{a} \mathrm{~B}^{b}$ & $\mathrm{NP}^{c} \mathrm{~F}^{c}$ \\
$r_{\mathrm{ev}}$ & $3.0 \sigma$ & $1.0 \varepsilon$ & $10.0 \varepsilon$ \\
$r_{\mathrm{c}}$ & $2.5 \sigma$ & $1.5 \sigma$ & $1.5 \sigma$ \\
${ }^{a}$ Nanoparticles. ${ }^{b}$ Backbone beads. ${ }^{c}$ Functionalized beads. &
\end{tabular}

The polymer systems under such temperature, roughly twice the glass transition temperature $T_{\mathrm{g}}$ of the polymers, are expected to be in melt state. ${ }^{53}$ Since our aim is to investigate the processing behavior of PNCs under shear, such a setting is necessary.

The simulations were started from a non-overlapped configuration of NPs and functionalized chains in a large simulation box implementing periodic boundary conditions. The simulation box was gradually compressed to a volume $V$ during the initialization phase of the simulation until a reasonable polymer melt-like density of $\rho_{\text {poly }}=n_{\text {bead }} m / V_{\text {poly }}=$ $0.85 \mathrm{~m} / \sigma^{3}$ was obtained, where $n_{\text {bead }}$ being the number of beads inside the system and $V_{\text {poly }}$ is the volume of the simulation box occupied by the polymer only but not the NPs. In the current study, large system sizes composed of $n_{\mathrm{NP}}=40-100 \mathrm{NPs}$ and $n_{\text {chain }}=200$ polymer chains are utilized. Thus, the volume fraction of the NPs, defined by $\phi_{\mathrm{NP}}=n_{\mathrm{NP}} \pi \sigma_{\mathrm{NP}}{ }^{3} /(6 V)$, is varied from 0.08 to 0.18 .

Then, we ran the simulations in a canonical (NVT) ensemble over 200 million time steps for equilibrium. After equilibrium was reached, steady shear flow was applied to these systems through a constant triclinic deformation in LAMMPS with particle velocities remapped when a shear-gradient boundary is crossed. This implementation is equivalent to the classic LeesEdwards boundary conditions. ${ }^{57}$ By this method we can impose a linear velocity profile with constant gradient $\dot{\gamma}=\partial v_{x} / \partial y$. The flow direction is parallel to the $x$-axis, the velocity gradient is along the $y$-axis, and the $z$-axis refers to the vorticity direction. During the shearing process, the stress tensor can be calculated using the virial theorem:

$$
\sigma_{\alpha \beta}=\frac{1}{V}\left[\sum_{i=1}^{n} m_{i} v_{i \alpha} v_{i \beta}+\sum_{i=1}^{n-1} \sum_{j=i+1}^{n} r_{i j \alpha} F_{i j \beta}\right]
$$

where $\alpha$ and $\beta$ take on values $x, y, z$ to generate the 6 components of the symmetric tensor, $n$ is the total number of particles $\left(n=n_{\mathrm{NP}}+n_{\text {bead }}\right), m_{i}, v_{i \alpha}$, and $v_{i \beta}$ are the mass and $\alpha$ - and $\beta$ component velocities of particle $i$, respectively, $r_{i j \alpha}$ and $F_{i j \beta}$ are the $\alpha$-component separation distance and $\beta$-component force acting between particle $i$ and $j$, respectively. To obtain reliable results, five independent simulations were performed for each system. Each simulation upon the presence of shear consisted of 250 million steps to guarantee the steadiness followed by 50 million steps to sample the properties of interest.

\section{Results and discussion}

\subsection{Shear-induced $1 D$ alignment of NP strings}

We start to simulate a system with functionalization degree $d_{\mathrm{F}}=$ 0.12 and filler volume fraction $\phi_{\mathrm{NP}}=0.15$. We found that when under the quiescent condition, the NPs can spontaneously selfassemble into interconnected strings (Fig. 1c). We then analyze the effect of shear on such a system by varying the shear rate $\dot{\gamma}$ from $10^{-5}$ to $2 \times 10^{-2} \tau^{-1}$. The steady-state structures of the NPs after shear are displayed in Fig. 2. It is clear that the initial interconnected strings can be broken up and restructured by shear force. When a moderate shear rate (e.g., $\dot{\gamma}=5 \times 10^{-4} \tau^{-1}$ or $2 \times 10^{-3} \tau^{-1}$ ) was applied, the NPs can arrange into $1 \mathrm{D}$ strings 


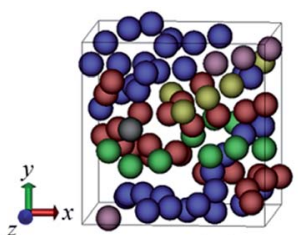

(a) $10^{-5} \tau^{-1}$

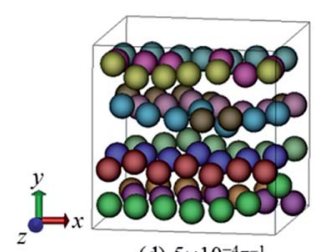

(d) $5 \times 10^{-4} \tau^{-1}$

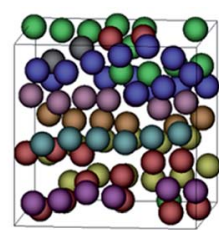

(b) $5 \times 10^{-5} \tau^{-1}$

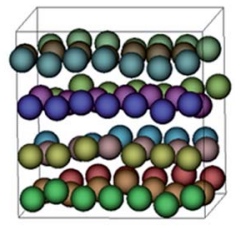

(e) $2 \times 10^{-3} \tau^{-1}$

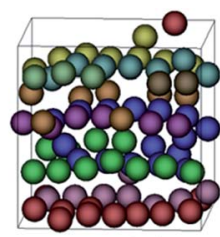

(c) $10^{-4} \tau^{-1}$

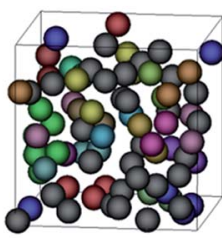

(f) $2 \times 10^{-2} \tau^{-1}$
Fig. 2 Steady-state configurations of NPs obtained at different shear rates $\dot{\gamma}$. The gray spheres denote individual NPs, while other spheres represent the NPs belonging to clusters (different clusters are shown in different colors). Because of the periodic boundary conditions of the simulation box, one can see that some clusters are located in both sides of the boundary regions. The $x_{-}^{-}, y^{-}$, and $z$-axes refer to velocity, velocity gradient and vorticity directions, respectively.

along the flow $(x)$ direction (Fig. 2d and e). Keeping on increasing the shear rate $\left(\dot{\gamma}=2 \times 10^{-2} \tau^{-1}\right)$, the strings would finally be broken into short strings or even individual NPs, as shown in Fig. $2 \mathrm{f}$.

In order to describe the NP organization quantitatively, we calculated the probability distribution of the nearest neighbor coordination numbers of the NPs. Here the nearest neighbor coordination number was defined as follows: for the NPs in a simulation system, taking one of them as the central NP, the coordination number is the number of other NPs around the central NP within a distance of $4.5 \sigma$ (range of strong short-range attraction between the NPs). The results are presented in Fig. 3 . In the quiescent state, the majority of NPs have two neighbors and a small part of NPs have one or three neighbors, which indicates that the NPs were organized into interconnected strings because the NPs at the string ends, in the strings, and at the positions connecting different strings have one, two, and three neighbors, respectively. When the shear rate $\dot{\gamma}=5 \times 10^{-5}$ to $2 \times 10^{-3} \tau^{-1}$, the probability at two neighbors is increasing while the probability at

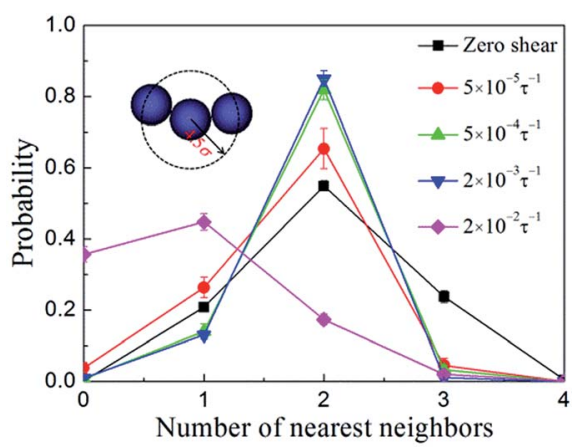

Fig. 3 Probability distribution of the nearest neighbor (defined to be closer than $4.5 \sigma$ ) coordination numbers of NPs at different shear rates $\dot{\gamma}$. The inset is the schematic diagram showing the definition for the number of nearest neighbors. three neighbors decreases to a value close to zero, indicating that the interconnected strings were broken up and reconstructed into individual strings. With the shear rate further increasing (i.e., $\dot{\gamma}=$ $2 \times 10^{-2} \tau^{-1}$ ), the individual strings would finally be broken into much shorter strings or even separated NPs, which can be reflected from the decrease of the probability at two neighbors as well as the increase of the probabilities at zero and one neighbor. These results are consistent with that presented in Fig. 2.

Next, the sizes and number of the NP clusters in each system were also calculated. Here we defined a NP belonging to a cluster if its center of mass is within $4.5 \sigma$ from at least one other NP from that cluster. We found that under the quiescent condition, a main cluster, containing approximately $85 \%$ of NPs in the system, was formed, meaning that the majority of NP strings were connected to form network span throughout the system. When subjected to shear, the network would be broken into small clusters. As shown in Fig. 4a, the dependence of the largest and mean sizes as well as the number of clusters on the shear rate can be roughly divided into three regions, which relate to different packing structures shown in Fig. 2. Region 1 corresponds to the weak shear rate with $\dot{\gamma}<10^{-4} \tau^{-1}$, where the largest (mean) cluster size decreases dramatically, and consequently, the number of clusters increases rapidly, in accordance with the phenomenon that the interconnected NP strings were gradually broken into separated strings as shown in Fig. 2a-c. While in the intermediate region $10^{-4} \tau^{-1}<\dot{\gamma}<3 \times 10^{-3} \tau^{-1}$ (Region 2), the sizes and number of clusters only show slight variations. In this region, the stabilized structures were formed. Finally, in Region $3\left(\dot{\gamma}>3 \times 10^{-3} \tau^{-1}\right)$, the cluster sizes further decrease and the number of clusters steeply increases, where the long strings would be further broken up by the shear flow.

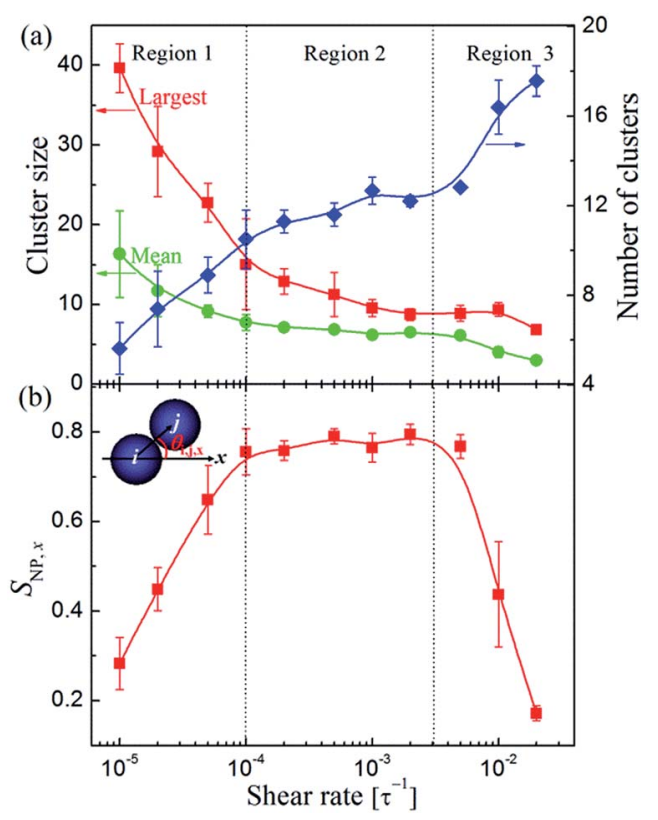

Fig. 4 (a) The largest and mean cluster sizes (left axis) and number of clusters (right axis) in systems at different shear rates $\dot{\gamma}$. (b) The second Legendre polynomial $S_{N P, x}$ of NP clusters as a function of shear rate $\dot{\gamma}$. The inset is the schematic diagram illustrating the definition of the angle between the particle $i$ to $j$ vector and flow direction ( $x$-axis), $\theta_{i, j, x}$. 
Moreover, we have also quantified the alignment of the NP clusters via the second Legendre polynomial $S_{\mathrm{NP}, x}$, also known as the Hermans orientation function, which was defined as follows:

$$
S_{\mathrm{NP}, x}=\frac{1}{\sum_{i=1}^{n_{\mathrm{NP}}} n_{\mathrm{c}}(i)}\left\{\sum_{i=1}^{n_{\mathrm{NP}}} \sum_{j=1}^{n_{\mathrm{c}}(i)}\left[\frac{1}{2}\left(3 \cos ^{2} \theta_{i, j, x}-1\right)\right]\right\}
$$

where $n_{\mathrm{NP}}$ is the number of NPs in the system, $n_{\mathrm{c}}(i)$ is the number of the nearest neighbors to particle $i$ and $\theta_{i, j, x}$ is the angle between the particle $i$ to $j$ vector and the $x$-axis. The order parameter $S_{\mathrm{NP}, x}$ takes unity when all pairs of neighboring NPs are aligning along the flow direction ( $x$-axis) and zero when they take random orientations. Fig. $4 \mathrm{~b}$ displays the results of the second Legendre polynomial $S_{\mathrm{NP}, x}$ at different shear rates. We find that the order parameter $S_{\mathrm{NP}, x}$ can also be divided into three regions consistent with the results in Fig. $4 \mathrm{a}$. The $S_{\mathrm{NP}, x}$ first increases in Region 1 but finally decreases in Region 3. While in Region 2, there is a platform on which $S_{\mathrm{NP}, x}$ is close to 0.8 , indicating that in the intermediate shear-rate region (ranging from $10^{-4} \tau^{-1}$ to $3 \times 10^{-3} \tau^{-1}$ ), highly oriented NP strings were formed.

On the basis of the above analyses, we showed that there is a threshold shear rate, which turns out to be $\dot{\gamma}_{\text {thr }} \approx 10^{-4} \tau^{-1}$, below which no 1D alignment can be achieved. Meanwhile, we also showed that when the shear rate exceeds a critical value, $\dot{\gamma}_{\mathrm{c}}$ $\approx 3 \times 10^{-3} \tau^{-1}$, the well-defined structures would finally be broken into randomly oriented short strings or even individual NPs. In the intermediate region, i.e., $\dot{\gamma}_{\text {thr }}<\dot{\gamma}<\dot{\gamma}_{c}$, the shear flow will direct the formation of long strings aligning along the shear direction. We found that the velocity-gradient and gradientvorticity planes often show sliding layer (Fig. 5a) and hexagonal lattice (Fig. 5b) formations, respectively, and each layer composed of almost the same number of strings (Fig. 5c), indicating the well-defined ordering of the NPs in the system. Such a shear-induced emergence of aligned strings at moderate shear followed by a disordered state at high shear has been previously observed for sheared colloidal suspensions that consist of soft, ${ }^{29,58,59}$ hard,${ }^{60}$ or charged ${ }^{61}$ particles. These strong similarities in the dynamic behavior are quite surprising since the investigated systems have rather different structural properties. Hence, there might be some (non-trivial) physical connection between these systems, which would be an interesting point requires to explore further.
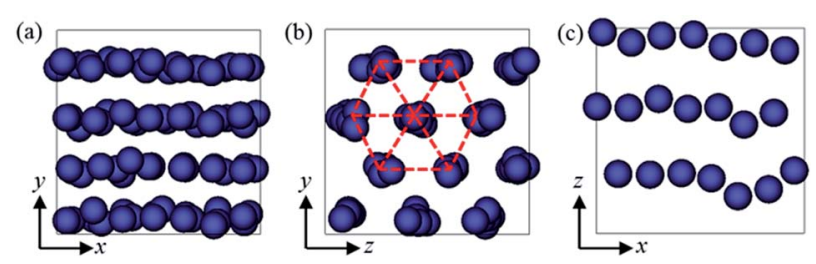

Fig. 5 Structures formed at shear rate $\dot{\gamma}=2 \times 10^{-3} \tau^{-1}$. (a) Front view (perpendicular to $x y$ plane) and (b) side view (perpendicular to $y z$ plane). (c) A slice of the simulation box in $x z$ plane. The $x-, y-$, and $z-$ axes refer to velocity, velocity gradient and vorticity directions, respectively.
In light of the above interesting structures formed at moderate shear rates, we are curious to know whether such wellordered structure can be observed in other similar systems. To achieve that, we should choose systems initially (in the quiescent state) with spontaneously formed NP strings. In our past study, ${ }^{18}$ we have showed that the configuration of NP assemblies in quiescent system is determined by $d_{\mathrm{F}} / \phi_{\mathrm{NP}}$. When $d_{\mathrm{F}} / \phi_{\mathrm{NP}}$ is ranged from 0.72 to 1.56 , the NPs would organize into stringlike assemblies. Therefore, in the current study, three other systems with $d_{\mathrm{F}}=0.06$ and $\phi_{\mathrm{NP}}=0.08, d_{\mathrm{F}}=0.09$ and $\phi_{\mathrm{NP}}=0.12$, and $d_{\mathrm{F}}=0.15$ and $\phi_{\mathrm{NP}}=0.18$, were selected. All the results are illustrated in Fig. 6, where the result for the previous system with $d_{\mathrm{F}}=0.12$ and $\phi_{\mathrm{NP}}=0.15$ is also included for comparison. Since $d_{\mathrm{F}} / \phi_{\mathrm{NP}}$ for these systems is ranged from 0.75 to 0.83 , the string-like assemblies of the NPs can indeed be observed (see Fig. 6a0-d0). Upon the imposing of moderate shear $(\dot{\gamma}=2 \times$ $\left.10^{-3} \tau^{-1}\right)$, the NPs were finally reorganized into 1D strings aligning along the flow direction (Fig. 6a1-d1), which would become much more ordered with increasing the NP concentration due to sliding effect between the layers (Fig. $6 \mathrm{c} 1$ and d1).

\subsection{Structural formation mechanism}

To understand the mechanism of forming the ordered NP structures under steady shear, we turn to analyze the structural, dynamic, and rheological properties of the equilibrated and sheared PNCs in the following. The discussions are only focused on the system with $d_{\mathrm{F}}=0.12$ and $\phi_{\mathrm{NP}}=0.15$, if there are no specific instructions.

We first monitored the detailed evolution process of NP morphologies during shear. Some typical results are shown in Fig. 7. We found that even at the lowest shear rate tested $(\dot{\gamma}=$ $\left.10^{-5} \tau^{-1}\right)$, the initial interconnected NP-string network can be broken up, as illustrated in Fig. 7a. At moderate shear rate (i.e., $\dot{\gamma}=2 \times 10^{-3} \tau^{-1}$ ), the initial network was broken into much smaller strings or individual NPs in a time $t<2 \times 10^{4} \tau$ (Fig. 7a, $\mathrm{b}$, and $\mathrm{c} 1$ ). Then, the shear flow induced the ordering of these small strings along the flow direction, and at the same time led to inter-string connections (Fig. 7a, b, and c2). The breaking and
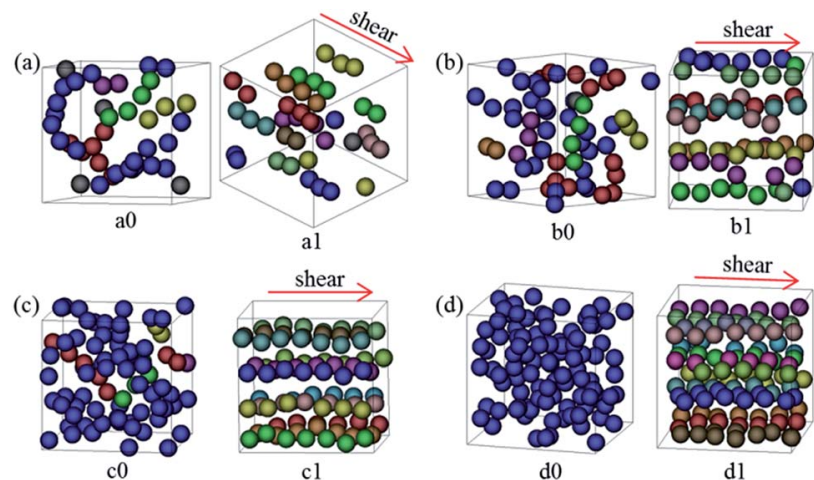

(d)
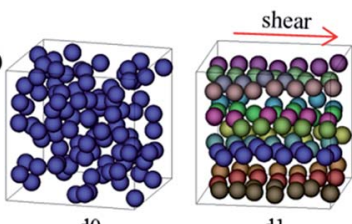

d1

Fig. 6 NP morphologies $(\mathrm{a} 0-\mathrm{d} 0)$ before and (a1-d1) after applying shear for the systems with (a) $d_{\mathrm{F}}=0.06$ and $\phi_{\mathrm{NP}}=0.08$, (b) $d_{\mathrm{F}}=0.09$ and $\phi_{\mathrm{NP}}=0.12$, (c) $d_{\mathrm{F}}=0.12$ and $\phi_{\mathrm{NP}}=0.15$, and (d) $d_{\mathrm{F}}=0.15$ and $\phi_{\mathrm{NP}}$ $=0.18$, where the shear rate $\dot{\gamma}=2 \times 10^{-3} \tau^{-1}$. The meaning of spheres in various colors is same as Fig. 2 . 

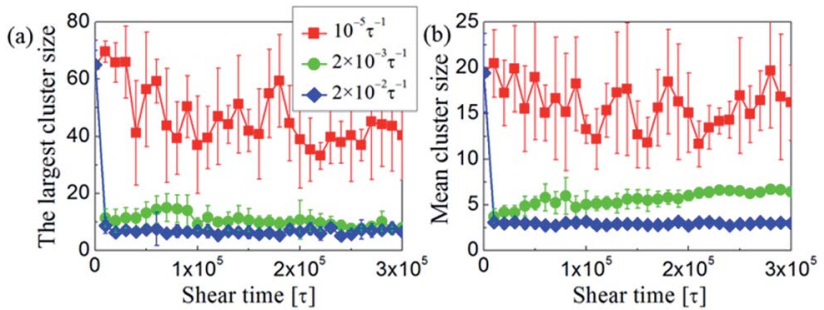

(c)

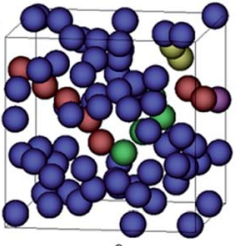

$\mathrm{c0}$

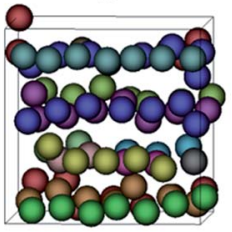

c3

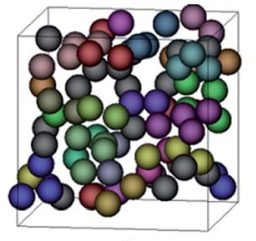

$\mathrm{cl}$

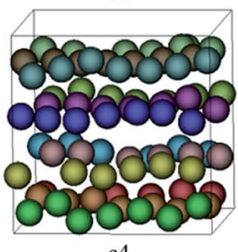

c4

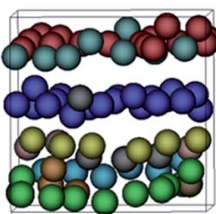

c2

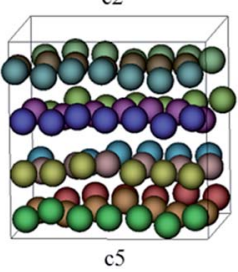

Fig. 7 (a) The largest and (b) mean cluster sizes as a function of time $t$ at three typical shear rates $\dot{\gamma}$. (c) Typical snapshots of NP strings sheared at $\dot{\gamma}=2 \times 10^{-3} \tau^{-1}$ for different time: (c0) $t=0$, (c1) $2 \times 10^{4}$ (c2) $8 \times 10^{4}$, (c3) $1.5 \times 10^{5}$, (c4) $2.5 \times 10^{5}$, and (c5) $3 \times 10^{5} \tau$. The meaning of spheres in various colors is same as Fig. 2.

recombining of NP strings would occur for many times until a steady-state structure was obtained for which the individual NP strings highly aligned along the shear direction were formed (Fig. 7a, b, c4 and c5). At high shear rate (i.e., $\dot{\gamma}=2 \times 10^{-2} \tau^{-1}$ ), the interconnected NP-string network would also be broken into small strings or individual NPs in the initial short time $(t<2 \times$ $\left.10^{4} \tau\right)$. However, these small strings or individual NPs are unable to coarsen into larger strings again (Fig. 7a and b) due to the extremely high shear force. For the above processes, the effect of shear on the NP morphologies can be quantitatively discussed by using the Péclet number, $\mathrm{Pe}=d_{\mathrm{NP}}{ }^{2} \dot{\gamma} / 4 D_{\mathrm{NP}}$, where $D_{\mathrm{NP}}$ is the NP diffusion coefficient obtained through $6 D_{\mathrm{NP}} t=\Delta r^{2}(t)$, and $\Delta r^{2}(t)$ is the mean-squared displacement of NPs. For $\dot{\gamma}=$ $10^{-5} \tau^{-1}$, we got $\mathrm{Pe}=7.81>1$. The result indicates that the shear-induced drag force on a NP is higher than the random force (due to the thermal fluctuations) on it. Therefore, the flow can alter the NP dispersion state even at the lowest shear rate tested $\left(\dot{\gamma}=10^{-5} \tau^{-1}\right)$.

It should be noted that the NP strings in the systems were also bridged through the functionalized polymer. Therefore, the changes of polymer structures and dynamics should also play an important role in the alignment of the NP strings. On this basis, we then began to investigate the structures and dynamics of the polymer under shear. Fig. 8 shows the diagonal components of the gyration tensor $R_{\alpha \alpha}$ as a function of shear rate. We found that the polymer chains were almost fully isotropic at rest (not shown). However, as shear was applied, the chains would elongate along the flow $(x)$ direction, and at the same time, slightly contract along the gradient $(y)$ and vorticity $(z)$ direction

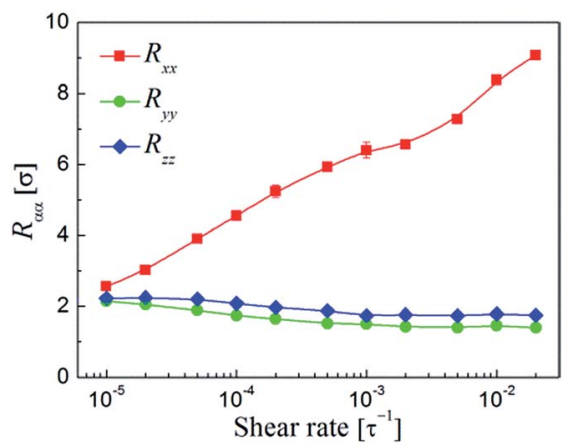

Fig. 8 Diagonal components of the gyration tensor $R_{\alpha \alpha}$ of the functionalized polymer along the flow $(x)$, gradient $(y)$, and vorticity $(z)$ directions of the shear flow as a function of shear rate $\dot{\gamma}$.

with $R_{y y}<R_{z z}$ throughout. It is clear that the shear in these systems, even at the lowest rate studied $\left(\dot{\gamma}=10^{-5} \tau^{-1}\right)$, is substantial enough that the polymer chains became deformed from their equilibrium configurations. Such tendencies can be described by the dimensionless Weissenberg number, $\mathrm{Wi}=\dot{\gamma} \tau_{1}$, where $\tau_{1}$ is the longest relaxation time of polymer. Theoretical considerations based on the Rouse model predict that $\tau_{1}$ follows the relation $\tau_{1}=2 R_{\mathrm{g}, 0}{ }^{2} / \pi^{2} D_{\text {poly }}{ }^{62}$ where $R_{g, 0}$ is the radius of gyration of polymer at equilibrium and $D_{\text {poly }}$ is the polymer diffusion coefficient. For the simulations performed, we found $\tau_{1}=1.74 \times 10^{-5} \tau$ which yields values for Wi between $1.74-3840$ for the entire shear rate range investigated. Clearly, since the polymer chains deform much more rapidly than relax, the polymer can be elongated along the flow direction.

The elongation of the polymer chains will lead to the fact that the network structures of the PNCs become distorted or broken. To better understand the effect of shear on the polymerbridged NP-string network, we quantified the alignment of the chain bridges between two neighboring adsorbing (functional) sites via the second Legendre polynomial $S_{\text {bridges }, \alpha \text { : }}$

$$
S_{\text {bridges }, \alpha}=\frac{1}{n_{\text {chain }}\left(N_{\mathrm{F}}-1\right)}\left\{\sum_{i=1}^{n_{\text {chain }}} \sum_{j=1}^{N_{\mathrm{F}}-1}\left[\frac{1}{2}\left(3 \cos ^{2} \theta_{j, j+1, \alpha}-1\right)\right]\right\}
$$

where $n_{\text {chain }}$ is the number of polymer chains, $N_{\mathrm{F}}$ is the number of functionalized beads in a chain, $\theta_{j, j+1, \alpha}$ is the angle between the end-to-end vector ( $j$ to $j+1$ functional site in a chain) and the Cartesian axes $\alpha \in\{x, y, z\}$. The results are shown in Fig. 9a. At the lowest shear rate studied, $S_{\text {bridges }, x}, S_{\text {bridges }, y}$ and $S_{\text {bridges }, z}$ are almost at zero, indicating that the chain bridges do not have any preferred orientation. As the shear rate is increasing, $S_{\text {bridges }, x}$ is increased to about 0.6 and at the same time $S_{\text {bridges, } y}$ and $S_{\text {bridges, } z}$ are decreased to about -0.3 , which means that the bridges began to align along the flow direction. Furthermore, it is apparent that the upper bound of 1 for $S_{\text {bridges }, x}$ is not reached because of the thermal fluctuations and entanglements between the polymer chains and NPs. We should note that in the current study polymer chains are connected by NPs to form networks. We anticipated that when the networks deform to break under shear, the polymer bridges in these networks 


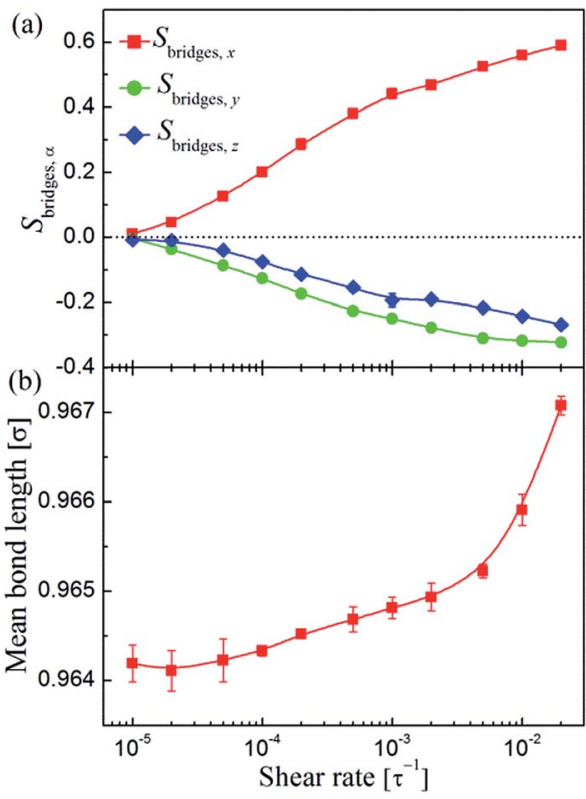

Fig. 9 (a) The second Legendre polynomial $S_{\text {bridges, } \alpha}$ of the chain bridges between two neighboring adsorbing (functional) sites along the flow $(x)$, gradient $(y)$, and vorticity $(z)$ directions. (b) Mean bond length of the polymer as a function of shear rate $\dot{\gamma}$.

would slight stretch. Fig. 9b shows the mean bond length as a function of shear rate. When the shear rate was weak $(\dot{\gamma}<$ $\left.10^{-4} \tau^{-1}\right)$, the bond length maintained at equilibrium value $\left(l_{0}\right.$ $\approx 0.9642 \sigma$ ) indicating the absence of chain stretching. While at high shear, especially when $\dot{\gamma}>3 \times 10^{-3} \tau^{-1}$, chain stretching indeed can be observed. It should be noted that the bond stretching was below $0.5 \%$ for the highest flow strengths applied, indicating that our simulations did not include any unphysical simulation artifacts. ${ }^{63}$

The stretching of the polymer bridges may lead to the debonding of the functionalized beads on the NPs, and thus result in the breaking of the polymer-bridged NP network. To gain more details about this issue, we then calculated the density profile of the functionalized beads around the NPs (the pair distribution function $g_{\mathrm{NP}-\mathrm{F}}(r)$ ) and the total attraction energy between the NPs and functional sites $\left(E_{\mathrm{NP}-\mathrm{F}}\right)$. The corresponding results are presented in Fig. 10a and b, respectively. We find that the peaks of the $g_{\mathrm{NP}-\mathrm{F}}(r)$ are located at $r=2.62 \sigma$ (Fig. 10a), which indicates the tight adsorption of functionalized beads on the NPs. When $\dot{\gamma}$ below $10^{-4} \tau^{-1}$, the variations of both the $g_{\mathrm{NP}-\mathrm{F}}(r)$ and $E_{\mathrm{NP}-\mathrm{F}}$ are negligible. At moderate shear rates (i.e., $10^{-4} \tau^{-1}<\dot{\gamma}<3 \times 10^{-3} \tau^{-1}$ ), the peaks of the $g_{\mathrm{NP}-\mathrm{F}}(r)$ slightly decrease, and accordingly the absolute value of the $E_{\text {NP-F }}$ also shows a slight decrease, indicating the debonding of a small amount of functionalized beads from the NPs. Therefore, we anticipated that the threshold shear rate $\dot{\gamma}_{\text {thr }}$ for the formation of 1D NP-strings is also the shear rate at which the functionalized beads around the NPs begin be dragged by shear since they relax slower than shear. As the functionalized beads adsorbed on the NPs were dragged by the shear, the polymer bridges between the NPs should exhibit slight chain stretching
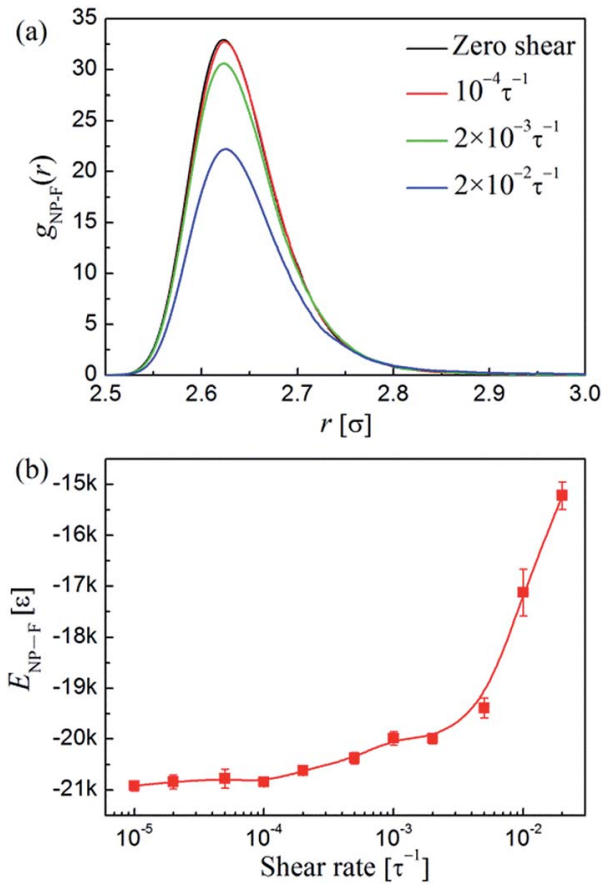

Fig. 10 (a) Pair distribution function of functionalized beads around the NPs, $g_{\mathrm{NP}-\mathrm{F}}(r)$, at different shear rates $\dot{\gamma}$. (b) Total attraction energy between NPs and functionalized beads, $E_{\mathrm{NP}-\mathrm{F}}$, as a function of shear rate $\dot{\gamma}$.

(Fig. 9b). While after $\dot{\gamma}$ exceeds $3 \times 10^{-3} \tau^{-1}$, the polymerbridged NP-string network became broken because the interfacial attractions between the NPs and functionalized beads cannot resist the retraction of polymer bridges between the NPs as they were significantly stretched at such high shear strength. Therefore, in this region, a large number of functionalized beads were dramatically breaking away from the NPs.

Finally, we investigated the rheological properties of the sheared PNC systems to better understand the shear-induced behavior. Fig. 11a shows the first and second normal stress differences, $N_{1}=\sigma_{x x}-\sigma_{y y}$ and $N_{2}=\sigma_{y y}-\sigma_{z z}$, respectively, as a function of shear rate. Apparently, the $N_{1}$ profile looks similar in shape to those of Fig. $9 \mathrm{~b}$ and $10 \mathrm{~b}$. The values of $N_{1}$ are positive, therefore, the microstructure of the PNCs can be elongated along the flow direction $\left(\sigma_{x x}>\sigma_{y y}\right)$. While the $N_{2}$ values are slightly negative; since $\sigma_{y y}<\sigma_{z z}$, the PNC structures would be compressed in the gradient $(y)$ direction, and thus $R_{y y}$ $<R_{z z}$ (Fig. 8) and $S_{\text {bridges, } y}<S_{\text {bridges }, z}$ (Fig. 9a). Fig. 11b shows the temporal evolution of shear stress $\sigma_{x y}$ during the startup of shear. It is apparent that when $\dot{\gamma}<10^{-4} \tau^{-1}$, the computed $\sigma_{x y}$ slowly increases as the shear is turned on until it converges to its steady state value. After $\dot{\gamma}$ exceeds $10^{-4} \tau^{-1}$, the temporal development of $\sigma_{x y}$ shows an initial stress overshoot and then gradually drops to a well-defined steady state value. This overshoot is closely related to the yielding and/or breaking of the polymer-bridged NP network. In Fig. 11c, we plot the steadystate shear stress $\sigma_{x y}$ as a function of shear rate $\dot{\gamma}$. We find that our data can be well fitted by Ostwald-de Wale ${ }^{64}$ power-law equation: 
(a)
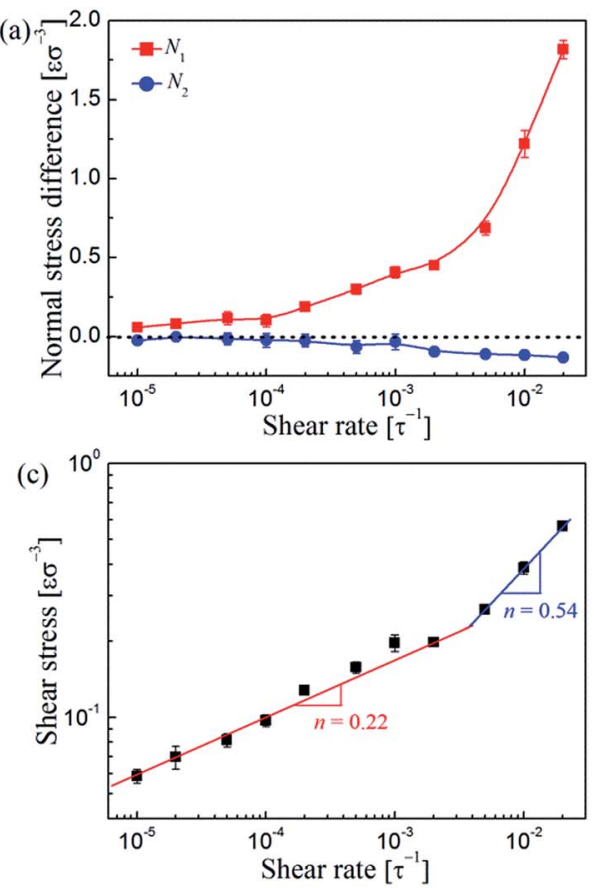

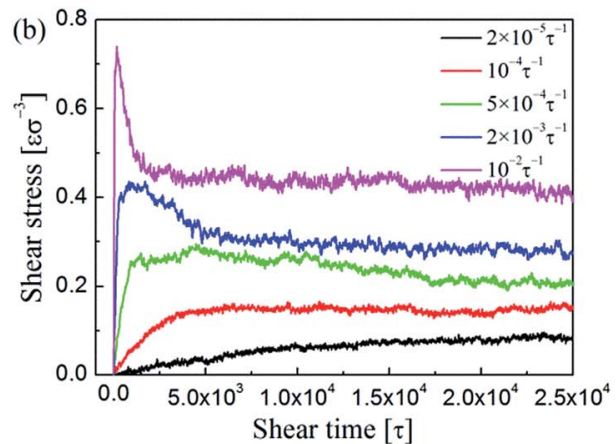

(d)

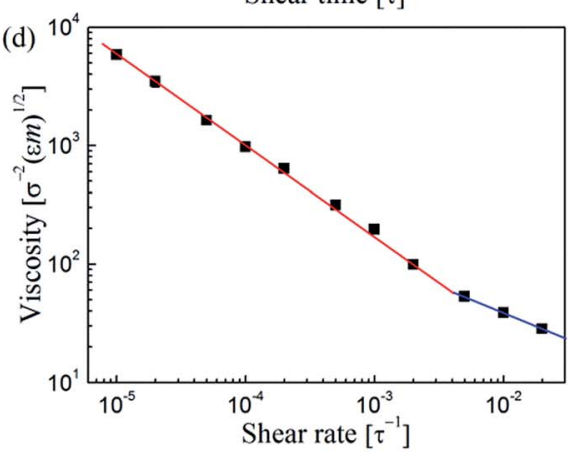

Fig. 11 (a) The first and second normal stress differences, $N_{1}$ and $N_{2}$, respectively, as a function of shear rate $\dot{\gamma}$. (b) Shear stress $\sigma_{x y} v s$. time $t$ during startup shear for the PNC system at various shear rates $\dot{\gamma}$. (c) Shear stress $\sigma_{x y}$ and (d) viscosity $\eta$ as a function of shear rate $\dot{\gamma}$. The symbols show the simulation results, while the lines depict the prediction of eqn (7) for (c) and eqn (8) for (d).

$$
\sigma_{x y}=K \dot{\gamma}^{n}
$$

where $K$ is the flow consistency index, and $n$ is the dimensionless index characterizing the type of the fluid ( $n<1$ pseudoplastic; $n=1$ Newtonian; $n>1$ dilatant). The shear rate dependence of the viscosity is then calculated through ${ }^{64}$

$$
\eta=\frac{\sigma_{x y}}{\dot{\gamma}}=K \dot{\gamma}^{n-1}
$$

The results are presented in Fig. 11d. When $\dot{\gamma}<3 \times 10^{-3} \tau^{-1}$, we determined the exponent $n$ in eqn (7) and (8) to be 0.22 . This pseudoplastic response is due to the strong shear-thinning behavior of the system, which is directly related to the gradual alignment of the individual NP strings and polymer chains. While when $\dot{\gamma}$ beyond $3 \times 10^{-3} \tau^{-1}, n$ is increased to 0.54 , indicating that the shear-thinning was reduced to some extent because in this region the NP structures are not so well-defined as those in the previous regions.

On the basis of the above analyses, the mechanism for forming the shear-induced ordered structures of the NPs can be generalized as follows. At low shear rate $\left(\dot{\gamma}<\dot{\gamma}_{\text {thr }} \approx 10^{-4} \tau^{-1}\right)$, when Pe $>1$, the shear can alter the NP dispersion state, and when Wi $>1$, the shear can deform the polymer chains. Since the functionalized beads relax faster than shear at such low shear rate, the shear affects the NP morphology and polymer configuration independently. When at moderate shear rate (i.e., $\dot{\gamma}_{\text {thr }}<\dot{\gamma}<\dot{\gamma}_{\mathrm{c}} \approx 3 \times 10^{-3} \tau^{-1}$ ), the shear becomes faster than the functionalized bead relaxation rate. In this situation, the linkage of the NPs and functionalized polymer makes the NPs and polymer chains move integrally during shear. Therefore, the polymer-bridged NP-string network should resist the imposed shear integrally by deforming, yielding, and subsequently breaking its interblock bonds. The resulting small assemblies should still be stretched and then oriented along the flow direction. During such process, parts of the assemblies should become combined together again. The breaking and coarsening of the polymer-bridged NP strings should occur for many times until the final steady-state structure is obtained where the NP strings in the assemblies are highly aligned along the flow direction. However, after shear rate exceeds a critical value $\left(\dot{\gamma}>\dot{\gamma}_{\mathrm{c}}\right)$, the polymer bridges between the NPs would be dragged away from the NPs, and as a consequence, the initial polymer-bridged NP-string network would be broken up into very small structures, i.e., very short strings or individual NPs, and cannot coarsen into larger structures again at such high shear rate.

\subsection{The stability of the aligned strings after ceasing shear}

To explore the stability of the shear-induced aligned NP strings, the imposed shear flow is ceased and thereby the relaxing process of the nanocomposites can be investigated. Fig. 12a presents the morphologies of the sheared structures at different relaxing time for the system with $d_{\mathrm{F}}=0.12$ and $\phi_{\mathrm{NP}}=0.15$. It is interesting to find that the emerging structures remain stable after the cessation of shear. Fig. 12b also shows that only a very small decrease of $S_{\mathrm{NP}, x}$ can be identified in the relaxing stage, even when the relaxing time reaches to $2 \times 10^{5} \tau$ at which the polymer chains are almost recovering to isotropic 


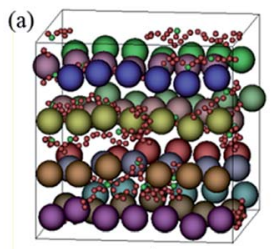

a0

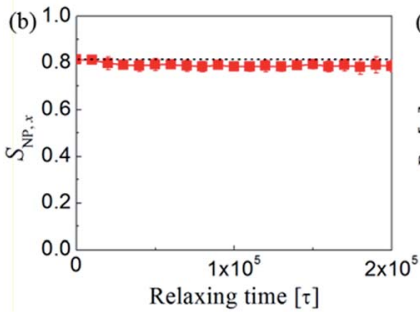

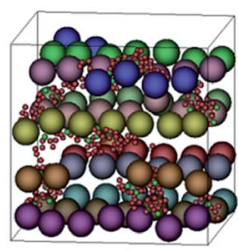

al

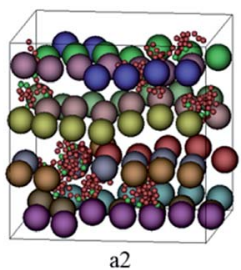

a2

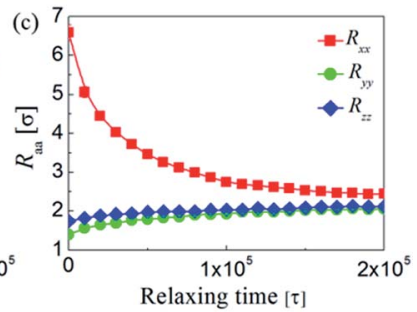

Fig. 12 (a) Typical snapshots of NP morphologies after ceasing the shear for different time: (a0) $t=0$, (a1) $10^{5}$, and (a2) $2 \times 10^{5} \tau$. The meaning of spheres in various colors is same as Fig. 2. Here, we also plotted five chains, which are denoted by small spheres in red (backbone beads) and green (functionalized beads) colors, to illustrate the relaxation of polymer chains. (b) The second Legendre polynomial $S_{N P, x}$ of NP strings and (c) diagonal components of the gyration tensor $R_{\alpha \alpha}$ of the functionalized polymer along the flow $(x)$, gradient $(y)$, and vorticity $(z)$ directions of the shear flow as a function of relaxing time.

configurations (Fig. 12c). The reason may be due to the fact that the motion of the NPs is strongly constrained by the surrounding matrix while the polymer chains still can relax to form new equilibrium structure, and as a result, the aligned NP strings can be maintained. This interesting finding suggests that stabilized 1D alignment of NP strings in polymer matrix can be achieved when applying an appropriate steady shear to specific functionalized polymer/NP systems, and the emerging structures can remain stable after the cessation of shear which ensures the applicability of shear-induced alignment.

\section{Conclusions}

In this work, we performed CGMD simulations to investigate the response of functionalized polymer/NP composites under steady shear. The uniqueness of the present modeling systems is that the functionalized polymers can induce the isotropically interacting spherical NPs spontaneously self-assemble into string-like superstructures, where the backbones of the functionalized chains inherently dislike the NPs but the periodically spaced functional groups on the chains are designed to show specific affinity for the NPs. The application of steady shear can lead to the breaking and rearrangement of these anisotropic NP assemblies. When shear rate below the threshold rate $\dot{\gamma}_{\text {thr }}$, the NP dispersion state can be altered if $\mathrm{Pe}>1$, but the NP strings do not flow align since functionalized beads adsorbed on the NPs relax faster than shear. In the intermediate shear-rate range $\left(\dot{\gamma}_{\text {thr }}<\dot{\gamma}<\dot{\gamma}_{\mathrm{c}}\right)$, the shear can induce 1D alignment to the NP strings, and the emerging structures can remain stable after ceasing the shear, which is required for many applications such as the fabrication of nanomaterial arrays. The formation of such well-defined structures reflects a balance among the NP mobility (Pe), polymer relaxation rate (Wi), NP-functional group interaction $\left(\varepsilon_{\mathrm{NP}-\mathrm{F}}\right)$, and external shear effect $(\dot{\gamma})$. While when shear rate exceeds a critical value $\dot{\gamma}_{c}$, the long NP strings would be broken into very short strings or even separated NPs because the functionalized polymers begin to lose their ability to direct NP assembly since they were seriously dragged away from the NPs at such high shear rate. It may be expected that the information derived in the present study provides a useful foundation for guiding the design and preparation of tailored PNCs.

\section{Acknowledgements}

This work was supported by the National Basic Research Program of China (2015CB654700 (2015CB674705)), Project of Department of Education of Zhejiang Province of China (GZ16551250002) and National Supercomputer Center in Guangzhou.

\section{References}

1 R. A. Vaia and J. F. Maguire, Chem. Mater., 2007, 19, 27362751.

2 S. K. Kumar and R. Krishnamoorti, Annu. Rev. Chem. Biomol. Eng., 2010, 1, 37-58.

3 M. E. Mackay, A. Tuteja, P. M. Duxbury, C. J. Hawker, B. Van Horn, Z. Guan, G. Chen and R. S. Krishnan, Science, 2006, 311, 1740-1743.

4 J. F. Moll, P. Akcora, A. Rungta, S. Gong, R. H. Colby, B. C. Benicewicz and S. K. Kumar, Macromolecules, 2011, 44, 7473-7477.

5 H. Deng, L. Lin, M. Ji, S. Zhang, M. Yang and Q. Fu, Prog. Polym. Sci., 2014, 39, 627-655.

6 N. Yousefi, X. Sun, X. Lin, X. Shen, J. Jia, B. Zhang, B. Tang, M. Chan and J. K. Kim, Adv. Mater., 2014, 26, 5480-5487.

7 M. Liu, Y. Du, Y.-E. Miao, Q. Ding, S. He, W. W. Tjiu, J. Pan and T. Liu, Nanoscale, 2015, 7, 1037-1046.

8 S. C. Glotzer and M. J. Solomon, Nat. Mater., 2007, 6, 557562.

9 Z. Nie, A. Petukhova and E. Kumacheva, Nat. Nanotechnol., 2010, 5, 15-25.

10 M. Grzelczak, J. Vermant, E. M. Furst and L. M. Liz-Marzán, ACS Nano, 2010, 4, 3591-3605.

11 J. Du and R. K. O'Reilly, Chem. Soc. Rev., 2011, 40, 2402-2416. 12 A. Walther and A. H. Müller, Chem. Rev., 2013, 113, 5194-5261. 13 P. Akcora, H. Liu, S. K. Kumar, J. Moll, Y. Li, B. C. Benicewicz, L. S. Schadler, D. Acehan, A. Z. Panagiotopoulos, V. Pryamitsyn, V. Ganesan, J. Ilavsky, P. Thiyagarajan, R. H. Colby and J. K. Douglas, Nat. Mater., 2009, 8, 354-359. 14 T. Nakano, D. Kawaguchi and Y. Matsushita, J. Am. Chem. Soc., 2013, 135, 6798-6801.

15 R. Shenhar, T. B. Norsten and V. M. Rotello, Adv. Mater., 2005, 17, 657-669.

16 T. Curk, F. J. Martinez-Veracoechea, D. Frenkel and J. Dobnikar, Nano Lett., 2014, 14, 2617-2622.

17 R. Klajn, M. A. Olson, P. J. Wesson, L. Fang, A. Coskun, A. Trabolsi, S. Soh, F. Stoddart and B. A. Grzybowski, Nat. Chem., 2009, 1, 733-738. 
18 L. Liu, C. Sun, Z. Li, Y. Chen, X. Qian, S. Wen and L. Zhang, Soft Matter, 2016, 12, 1964-1968.

19 V. Pryamtisyn, V. Ganesan, A. Z. Panagiotopoulos, H. Liu and S. K. Kumar, J. Chem. Phys., 2009, 131, 221102.

20 S. K. Kumar, N. Jouault, B. C. Benicewicz and T. Neely, Macromolecules, 2013, 46, 3199-3214.

21 B. Bozorgui, D. Meng, S. K. Kumar, C. Chakravarty and A. Cacciuto, Nano Lett., 2013, 13, 2732-2737.

22 D. Zhao, M. Di Nicola, M. M. Khani, J. Jestin, B. C. Benicewicz and S. K. Kumar, ACS Macro Lett., 2016, 5, 790-795.

23 A. Hirao, M. Hayashi, S. Loykulnant, K. Sugiyama, S. W. Ryu, N. Haraguchi, A. Matsuo and T. Higashihara, Prog. Polym. Sci., 2005, 30, 111-182.

24 L. Wu, Y. Wang, Y. Wang, K. Shen and Y. Li, Polymer, 2013, 54, 2958-2965.

25 J. F. Lutz, M. Ouchi, D. R. Liu and M. Sawamoto, Science, 2013, 341, 1238149.

26 F. S. Bates and G. H. Fredrickson, Phys. Today, 1999, 52, 3238.

27 V. Pelletier, K. Asakawa, M. Wu, D. H. Adamson, R. A. Register and P. M. Chaikin, Appl. Phys. Lett., 2006, 88, 211114.

28 J. M. Papalia, D. H. Adamson, P. M. Chaikin and R. A. Register, J. Appl. Phys., 2010, 107, 084305.

29 R. Scirocco, J. Vermant and J. Mewis, J. Non-Newtonian Fluid Mech., 2004, 117, 183-192.

30 J. Moll, S. K. Kumar, F. Snijkers, D. Vlassopoulos, A. Rungta, B. C. Benicewicz, E. Gomez, J. Ilavsky and R. H. Colby, ACS Macro Lett., 2013, 2, 1051-1055.

31 G. Allegra, G. Raos and M. Vacatello, Prog. Polym. Sci., 2008, 33, 683-731.

32 J. Liu, L. Zhang, D. Cao and W. Wang, Phys. Chem. Chem. Phys., 2009, 11, 11365-11384.

33 L. T. Yan and X. M. Xie, Prog. Polym. Sci., 2013, 38, 369-405.

34 H. Eslami, M. Rahimi and F. Müller-Plathe, Macromolecules, 2013, 46, 8680-8692.

35 C. M. Hadden, D. R. Klimek-McDonald, E. J. Pineda, J. A. King, A. M. Reichanadter, I. Miskioglu, S. Gowtham and G. M. Odegard, Carbon, 2015, 95, 100-112.

36 A. S. Pavlov and P. G. Khalatur, Soft Matter, 2016, 12, 54025419.

37 W. Li, B. Dong and L. T. Yan, Macromolecules, 2013, 46, 7465-7476.

38 M. Surve, V. Pryamitsyn and V. Ganesan, Langmuir, 2006, 22, 969-981.

39 P. S. Stephanou, V. G. Mavrantzas and G. C. Georgiou, Macromolecules, 2014, 47, 4493-4513.
40 G. Raos, M. Moreno and S. Elli, Macromolecules, 2006, 39, 6744-6751.

41 V. Pryamitsyn and V. Ganesan, J. Rheol., 2006, 50, 655-683. 42 J. D. Thomin, P. Keblinski and S. K. Kumar, Macromolecules, 2008, 16, 5988-5991.

43 V. Kalra, F. Escobedo and Y. L. Joo, J. Chem. Phys., 2010, 132, 024901.

44 E. Jaber, H. Luo, W. Li and D. Gersappe, Soft Matter, 2011, 7, 3852-3860.

45 Z. Pan, L. He, L. Zhang and H. Liang, Polymer, 2011, 52, 2711-2721.

46 J. T. Kalathi, G. S. Grest and S. K. Kumar, Phys. Rev. Lett., 2012, 109, 198301.

47 Y. Chen, L. Liu, Q. Yang, S. Wen, L. Zhang and C. Zhong, Langmuir, 2013, 29, 13932-13942.

48 Y. Chen, Z. Li, S. Wen, Q. Yang, L. Zhang, C. Zhong and L. Liu, J. Chem. Phys., 2014, 141, 104901.

49 F. Zhang, Y. Chen, C. Sun, S. Wen and L. Liu, RSC Adv., 2014, 4, 26706-26713.

50 S. Mortezapour, H. Eslami and E. N. Oskoee, J. Chem. Phys., 2015, 143, 034901.

51 L. Wang, Z. Zheng, T. Davris, F. Li, J. Liu, Y. Wu, L. Zhang and A. V. Lyulin, Langmuir, 2016, 32, 8473-8483.

52 Z. Zheng, J. Shen, J. Liu, Y. Wu, L. Zhang and W. Wang, RSC $A d v .$, 2016, 6, 28666-28678.

53 K. Kremer and G. S. Grest, J. Chem. Phys., 1990, 92, 50575086.

54 J. D. Weeks, D. Chandler and H. C. Anderson, J. Chem. Phys., 1971, 54, 5237-5247.

55 A. Schröder, M. Klüppel and R. H. Schuster, Macromol. Mater. Eng., 2007, 292, 885-916.

56 S. Plimpton, J. Comput. Phys., 1995, 117, 1-19.

57 A. W. Lees and S. F. Edwards, J. Phys. C: Solid State Phys., 1972, 5, 1921-1929.

58 A. Nikoubashman, G. Kahl and C. N. Likos, Phys. Rev. Lett., 2011, 107, 068302.

59 A. Nikoubashman, G. Kahl and C. N. Likos, Soft Matter, 2012, 8, 4121-4131.

60 J. Michele, R. Pätzold and R. Donis, Rheol. Acta, 1977, 16, 317-321.

61 S. R. Rastogi, N. J. Wagner and S. R. Lustig, J. Chem. Phys., 1996, 104, 9234-9248.

62 M. Doi and S. F. Edwards, in The Theory of Polymer Dynamics, Clarendon, New York, 1986.

63 A. Nikoubashman, R. L. Davis, B. T. Michal, P. M. Chaikin, R. A. Register and A. Z. Panagiotopoulos, ACS Nano, 2014, 8, 8015-8026.

64 J. D. Neves, M. V. da Silva, M. P. Gonçalves, M. H. Amaral and M. F. Bahia, Curr. Drug Delivery, 2009, 6, 83-92. 